\title{
Síndrome nefrótico en el primer año de vida
}

\author{
Colomba Norero V. ${ }^{*^{*}}$; M. Angela Delucchi B. ${ }^{2 *} ;$ M. Angélica Contreras M. ${ }^{3}$; \\ Elizabeth Lagos R. ${ }^{{ }^{*}}$; Pía Rosati M.. ${ }^{1 *}$; Vilma Nazal Ch. ${ }^{3}$; Martina Verdaguer D. ${ }^{3}$
}

\section{Nefrotic syndrome in the first year of life}

Among 99 children exrolled to c chilean muliceritic protocol on nephrotic symdrome along a 30 months pericd, eight polienls (five cir's) ore reported which were ycunger tho- 1 year of age tmeáion 7.5 monlhsị. C inica' 5 ymploms begur betore age 10 days if three of them, mic'oscooic hematuia was observed in sever; anterial hyperlension and acule re-al foilure were rescechively faund in four cases eacr. aboratory findings in nepr.rotic inton's wele nol diterent from those in older chileren with the syridrome. Kncwir causes of seconcary neohrotic syndrome were discarded in these palients. Histopathological siucies were done in six cases and showed difuse nesangual sclerosis $(n=2)$ tir one nount it was ossociated to Drash's syndromej; f:nnish rype in $=2 k$; foca segmental gomerulosclerosis

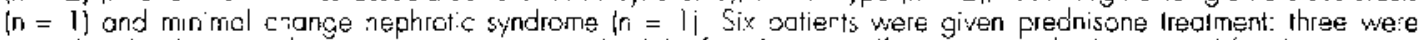
considered to be seroid resistant ione fotient each of the "innish ive. d ffuse mesancial selerosis and foca! segmenlai nephroselerssis\}: twc were steroc cependent ione without kiofsy and orother one with minimal change disease]. and one was steroid responisive. Cyslophasphamide was used in lwo patienis tmininal change and locol segmental nephrosclerosis! w th a iavousable outcome. Severe basterial inlections were soserved in seven and inrombosis in two ateced intonts. Four palents died. two had diffuse mesangio sclerosis \{ticth of sepric shock\} and the other two the firnish ype tone of pulmonay crtery thrombosist, in all of then clincat cisease tegun belore age four months. Cur:ently four patienls 'errain alive, three are under remission 'olter 3637 cnd 39 mo-th follow-up tirre'; cnd one has persiset prote nurio jofter 17 months follow-up'. "rognosis is poor in catients with nephrole syndrome associaled 10 difiuse mesangial telerosis ar finist type begning before age four months Vephrotic infanls with focal segmenial nephrosclercsis and minimal change disease behave like older pediatric patients with this syndrome.

iKay words. reohrotic syndrome, infonls: finnish rype, mirimal change, focol segmenta nephrosclerosis, diffuse nesangial sclerosis.!

El síndrome nefrótico (SN) en el menor de un año forma parte de un heterogéneo grupo de alteraciones de diferentes etiología, evolución, características clínico-patológicas y pronóstico. Para fines prácticos el trastorno se clasifica como congénito o infantil, según si las manifestaciones clínicas aparecen entre el nacimiento y los tres primeros meses de vida. o lo hacen más tarde cn el curso del primer año de vida.

1. Universidad de Chile. Facultad de Medicina. Departamento de Pediatria Occidente: Unidad de Nefrologia. Hospital San Juan de Dios. SSM Occidente.

2. Universidad de Chile, Facultad de Medicina Deparamento de Pediasía Oriente: Unidad de Nefrología. Hospital Luis Cal vo Mackenna. SSM Oriente.

3. L'triversidnd de Chile. Fucuitad de Medicina, Departamenco de Pediatria Norte; Unidad de Nefrología, Hos. pital Rohero del Río, SSM Norte.

* Subvención Fondecyt 19405
El tipo finlandés es el más conocido de los síndromes nefróticos congénitos. Se caracteriza por dilataciones pseudoquísticas de los túbulos. Es muy frecuente en Finlandia (1 por 100000 recién nacidos) $)^{1,2}$, generalmente se hereda en forma autosómica recesiva. Se describen formas aisladas y familiares on otros grupos étnicos, son resistentes a esteroides e inmunosupresores. Su letalidad es de $100 \%$ a los dos años y la sobrevida es -n promedio- 7,6 meses ${ }^{3 .} 4$ Las complicaciones más imporlantes y las responsables de la letalidad son las infecciones bacterianas severas y las trombosis.

Otra entidad asociada a síndrome nefrótico en monores de un año es ta denominada esclerosis mesangial difusa ${ }^{5}$, que puede manifestarse en forma aislada o asociada a seudohermafroditismo masculino (sindrome de Drash) ${ }^{6}$. Se caracteriza morfologicamente por esclerosis del 
glomérulo e hipertrofia de los podocitos. Tiene una tendencia a aparecer en familas de un modo que sugiere transmisión autośnica recesiva. Generalmente se manifiesta después de la edad de tres meses, los pacientes no responden a tratamiento esteroidal o inmunosupresor y evolucionan hacia la insuficiencia renal crónica, que es la principal causa de su muerte, antes de los tres años de vida.

Aunque menos frecuentes también se registran en los niños menores de un año las otras causas de síndrome nefrótico idiopático, como la enfermedad por cambios mínimos, la glomeruloesclerosis focal y segmentaria y la glomerulonefritis membranosa, cuya respuesta al tratamiento esteroidal puede ser semejante a la de los niños de más edad.

El propósito de este análisis fue describir las características clínicas, los resultados de los exámenes de laboratorio, el aspecto histológico, tratamiento, complicaciones y letalidad en un conjunto de pacientes menores de un año de edal con síndrome nefrótico detectados en un estudio colaborativo chileno.

\section{Pacientes y Método}

En un estudio nacional multicéntrico que abarcó un período de tueinta meses, en el que participarun once unidades pediátricas. se registraron 99 pacientes de sindrome uefrútico. En ocho de ellos, gue constituyen cl motivo de este análisis, la enferınedad comenzó durante el primer año de vida. Los requisitos para el diagnóstico fueron edema. proteinuria mayos a $40 \mathrm{~m} \mathrm{~m}^{\prime} \mathrm{nl}^{2}$ - día e hipoproteinemia con albuminenia inferior a $2.5 \mathrm{~g} / \mathrm{dl}$.

Los estudios histológicos renales se realizaron en muestras obtenidas por biopsia $(n=4)$ o necropsia $(n=2)$, Jnediante microscopía de Iuz, inmunofluorescencia $y$ mieroscopía electrónica. la mayoría de ellos por el Dr. Helmar Rosenberg (Hospital Clínico de la Pontificia Universidad Calólica de Chile), Los restantes por el Dr. Guillermo Murray (Faculad de Medicina de la Universidad de Chile). Mediante las pruebas apropiadas se descartó que el síndrome nefrólico fuese secundario a sífilis, taxoplas. mosis e inmunodeficiencia.

\section{Resultados}

Los márgenes de la edad de los ocho niños, cinco mujeres, eran 2 días a 11 meses, mediana de 7,5 meses. Tres pacientes habían nacido antes de término. En un paciente la placenta era de tamaño mayor que el normal. Tres presentaron anasarca al inicio de la enfermedad. En tres casos el trastorno se expresó antes de los diez días de vida, en uno a los cuatro meses y en cuatro a los once meses. Siete niños afectados tuvieron hemaluria microscópica; cuatro hipertensión arterial e insuficiencia renal aguda. El indice de los promedios de proteinemia $\mathrm{y}$ albuminemia (g/dl) fue 3,8/1,6. El promedio de la creatinina plasmática fue de $0,83 \mathrm{mg} / \mathrm{d}$ l (márgenes 0,2 a 3.17).

El estudio histológico de los cuatro pacientes en que la enfermedad se manifestó antes de los cuatro meses de edad, mostró en dos lesiones características del síndrome nefrótico de tipo finlandés, en otros dos csclerosis mesangial difusa (en uno de éstos asociadas a seudohermafroditismo masculino o síndrome de Drash). De los restantes nifios, en quienes la enfermedad comenzó cerca de la edad de once meses, se obluvieron muestras para estudio histológico en dos, uno tenía lesión con cambios mínimos y el otro glomeruloesclerosis focal y segmentaria.

Se emplearon esteroides en seis casos. Dos de aquellos cuya enformedad comenzó antes de los cuatro meses (los otros dos no alcanzaron a recibir tratamiento) resultaron ser resistentes. De los cuatro cuyo síndrome nefrótico empezó a los once meses, lodos recibieron esteroides, dos resultaron ser dependientes a éstos, uno sensible y otro resistente. En un paciente resistente y otro dependiente a corticoesteroides se utilizó ciclofosfamida: la respuesta fue favorable en ambos casos, que posteriormente se hicieron sensibles a la prednisona (tabla).

Las infecciones bacterianas severas fueron las complicaciones más frecuentes presentándose en $7(87,5 \%)$ pacientes, destacando cinco episodios de septicemia, una peritonitis, tres ncumopatias $y$ dos pielonefritis. Otras complicaciones graves fueron las trombosis, observadas en dos pacientes (25\%), en la vena renal y en la arteria pulmonar respectivantente. La le talidad en el periodo de observación fuc de 50\%: todas las inuertes ucurrieron en pacientes que tenían menos de cuatro meses al comienzo de los síntomas: dos con esclerosis mesangial difusa. fallecieron por falla circulatoria de causa séptica; de los dos con lesiones del tipo finlandés, uno murió de trombosis de la arteria pulmonar y el otro de insuficiencia renal crónica terminal y septicemia al cabo de 36 meses de seguimiento. Los euatro niños cuyas manifesta- 
Tabla

Sindrome nef́rólico en menotes de un año. Diagnóstico y evoluciół

\begin{tabular}{|c|c|c|c|c|c|}
\hline Sexo & $\begin{array}{l}\text { Edad } \\
\text { inicio }\end{array}$ & $\begin{array}{c}\text { Tiempo en } \\
\text { control }\end{array}$ & $\begin{array}{c}\text { Histo]ogía } \\
\text { renal }\end{array}$ & $\begin{array}{c}\text { Tratamiento } \\
\text { estado }\end{array}$ & Respuesta \\
\hline Mujer & $2 d$ & 120 & finlaudós & 110 & fallece \\
\hline Varón & 50 & $14 \mathrm{~d}$ & EMD(Dosh) & no & fallece \\
\hline Mujter & $10 \mathrm{~d}$ & $31 \mathrm{~m}$ & finlardes & pred & $\mathrm{R} / \mathrm{fallece}$ \\
\hline Varón & $4 \mathrm{~m}$ & 8 In & EMI & pred & R/ fallece \\
\hline Varón & $11 \mathrm{n}$ & $36 \mathrm{~m}$ & SNCM & pred/cicl & $\mathrm{R} /$ vivo \\
\hline Mujer & $11 \mathrm{~m}$ & $17 \mathrm{~m}$ & - & pred & D/ vivo \\
\hline Mujer & $11 \mathrm{M}$ & $34 \mathrm{~m}$ & GNFS & pred/cicl & D/ vivo \\
\hline Mujer & II m & $37 \mathrm{n}$ & - & pred & S/ vivo \\
\hline
\end{tabular}

K: resislente; D: dependienle: S: sensible: EML: esclerosis mesangial difusa: SVCA: síndme nefrótico con cambios mínimos; GNFS: glomerulontritis focal y sementaria; pred; prednisona; cicl: ciclofostamida: Drash: síndrome de...

ciones clínicas comenzaron a la edad de 11 meses sobreviven, tres cstán en remisión con función renal normal al cabo de 36,37 y 39 meses de seguimiento, el otro tiene proteinutia persistente a los 17 meses de seguimiento

\section{Comentario}

El síndrome nefrótico en menores de un año ha sido considerado excepcional en nuestro medio, pero en este recuento colaborativo se registraton ocho casos. que colresponden a $8 \%$ de los pacientes incluidos. Desde una perspectiva patológica el trastorno es heterogéneo, ya que los casos obedecen a cuatro entidades de características histopatologicas distintas, con respuesta a tratamiento, evolución. y mortalidad diferentes, dependiendo de la edad de inicio de los sintomas.

El sindrome nefrótico de tipo finlandés es de herencia autosomica recesiva. Su diagnóstico se puede realizar antes del nacimiento por aumento de la alfa-fetoproteina en la sangre materna y el líquido amniólico. Sc caracteriza por la pérdida masiva intrauterina de proteinas, $90 \%$ de las cuales son albúmina. sin que necesariamente exista insuliciencia renal al detectarse la enfermedad. E1 hallazgo más constante es el aumonto del tamaño de la placenta, cuyo peso excede a $25 \%$ del correspondiente al recién nacido, $y$ es frecuente el parto prematuro ${ }^{7}$. El gen que produce la enfermedad ha sido localizado en el brazo largo del cromosoma 19, pero la patogénesis de la enfermedad aún es desconocida. Se piensa que ella afecta sólo al rinón y no se ha comunicado recurrencias después del trasplante renal.

A pesar que la esclerosis mesangial difusa se presenta con más frecuencia después de los tres noses de vida, también se describe en recićn nacidos. Los dos pacientes incluidos en esta casuística se diagnosticaron antes de la edad de cuatro meses, evolucionando con inporlante detcrioro de la función renal, pero faIleciendo, en ambos casos de infeccioncs bacterianas severas. En este tipo de afección, a diferencia de lo que sucede en el tipo finlandés, no se ha descrito placentomegalia, premalurez o bajo peso de nacimiento y en el examen histológico el compromiso glomerular es predominante, lo que causa precozmente la insuficiencia renal crónica. La asociación de esta nefropatia a seudohermafroditismo masculino (disgenesia gonadal XY) y tumor de Wilms, sugiere una anomalía embriogénica ocurtida antes de la dilerenciación de las estructuras renales y genitales. No sicmpre la tríada está presente, porque es posible que el tumor se manifieste más tarde, por esto se habla de sindrome de Drash completo 0 incompleto, dependiendo si se presentan todas o sólo dos de las características, incluyendo la nefropatía ${ }^{6.7}$. Hasta hace atgún tiempo los pacientes con síndrome nefrótico infantit fallecían antes de los seis meses de edad, pero hoy es posible prolongar su sobrevida corrigiendo el déficit de proteínas y la nutrición (lo que se 
consigue mediante albúmina endovenosa y apoyo nutricional intensivo precor), tratando agresivamente las complicaciones $y$ recurriendo a nefrectonía bilateral, diálisis peritoneal y trasplante renal temprano ${ }^{8}$.

Cuando el síndrome nefrótico infantil comienza antes de la edad de cuatro meses, suele asociarse con cambios histológicos de esclerosis mesangial difusa o del tipo finlandés. y el pronóstico para los pacientes es malo, porque fallecen precozmente de complicaciones infecciosas o tromboembólicas, en contraste con lo que ocurre cuando el síndrome nefrótico se expresa después de los seis meses de edad, cuando el curso de la enfermedad se comporta como en niños mayores, en cl conjunto de los cuales, al cabo de 18 meses de seguimiento, no hubo mortalidad $y$ las frecuencias de complicaciones fueron en $1,8 \%$ tromboembólicas, en $36 \%$ infecciosas y en $1,8 \%$ hipertensión artcrial ${ }^{9}$.

\section{Resumen}

En un estudio colaborativo de 99 niños con síndrome nefrótico en un periodo de 30 meses. ocho (5 mujeres) eran menores de un año (mediana 7.5 meses de cdad) y constituyen el motivo de esta comunicación: ninguno lenía lúes. toxoplasmosis o inmunodeficiencia; en tres la afección se expresó antes de los 10 días; siete tenían hematuria microscópica, cualro hipertension arterial e insuficiencia renal. Los hallazgos de laboratorio fueron similares a los encontrados en niños mayores. En seis casos se hizo examen histológico, que mostró esclerosis mesangial difusa en dos, asociada en uno de ellos a sindrome de Drash; síndrome nefrólico de tipo finlandés en otros dos; glomeruloesclerosis focal y segmentaria en uno y síndrome nefrótico a cambios mínimos en olro. Seis pacientes fucron tratados con prednisona: tres resultaron resistentes (uno de tipo finlandês, otro de esclerosis mesangial difusa y otro de glomerulocsclerosis focal y segmentaria); dos fucron dependientes (uno con cambios mínimos y otro sin biopsia) y uno era sensible. En dos casos se empleó ciclofosfamida: en uno a cambios mínimos $y$ en otro con glomeruloesclcrosis focal y segmentaria. Las complicaciones observadas fueron infecciones bacterianas graves en siete pa- cientes y trombosis en dos. Cuatro de estos niños fallecieron: en todos, los síntomas comenzaron antes de los cuatro meses de edad, dos tenían esclerosis mesangial difusa y dos el tipo fînlandés. En los cuatro sobrevivientes el síndrome nefrótico empez 6 a la edad de once meses. tres están en remisión a los 36,37 y 39 meses de seguitniento, el otro tiene proteinuria persistente al cabo de 17 meses de seguimiento. Los niños cuyo síndrome nefrótico se expresa antes del cuarto mes de vida tienen mal pronóstico, pues morfológicamente sus lesiones se relacionan con esclerosis mesangial difusa o el tipo finlandés y fallecen precozmente de complicaciones infecciosas severas. Los pacientes menores de un año con glomeruloesclerosis focal y segmentaria o cambios mínimos se comportan como los de edades posteriores.

(Palabras clave: sindrome nefrótico, lactante menor, tipo finlandés, cambios mínimos, glomeruloesclerosis focal y segmentaria, esclerosis mesangial difusa.)

\section{Referencias}

I. Hover 1 , Atelersom C: Congenital ncphrotic syndrome. Clinics in Perinatology 1981: 8: 333-345.

2. Riphola J. Hutsenet $N$, Hullow $N$ : Congenital and infantlle nephrotic syndrome. En: Pediarric Kidney Discases. Chester M, Edelman JR (ed). Second Ed, Little. Brown and Co, Boswn, 1992: 1291-J305.

3. Hurtenen $N$ : Congenital nephrotic syndrome of Tinnish type. Arch Dis Child 1976: $51: 344-348$

4. Methan $J$. Mawer $M$. Sibley $R$. Vernier $R$ : Congenital nephrotic syndrome: evolution of medical management and results of renal transplantation. J Pediatr 1984: I05: $549-556$

5. Habib R. Boir E. Hétérogénéite des syandromes oéphroriques a début précoce du nourrisson. Helv Paediatr Acra 1973; 28: $9 \mathrm{~J}$.

6. Dirtsh A, Shermat F, Harmann W, Btizard $R$ - A syndrome of psetdohemaphroditism. Wilms' iumor. hypertenrion and degenerative renal disease. J Pediatr 1970; 76: 585-593.

7. Habib R: Nephrotic syodrome in the lst year of life. Pediatr Nephrol 1993; 7: 347-353.

8. Holmberg C. Antikainem M. Ronnhwh K. Ala-Houtats M. Jalunko H: Management of congenjtal nephrotic syndrome of the Finnish type. Pediatr Nephrol 1995; 1: 87.93.

9. Noreri C, Delucthi A, Lages E, Rosuti P: Grupo Colaborativo Chileno de Estudio del Sindrome Nefrosico del Niño: Evaluación a 18 meses de dos csqucmas de tratamiento con predrusona en el cuadro inicial del síndrome neftósico ptimario del niño. Rev Med Chile (en prensa). 